\title{
Co-culture with bone marrow stromal cells protects PC12 neuronal cells from tumor necrosis factor- $\alpha$-induced apoptosis by inhibiting the tumor necrosis factor receptor/caspase signaling pathway
}

\author{
LI LI $^{1}$, JING WANG $^{2}$, LING TANG $^{1}$, XIN YU $^{3}$, YI SUI $^{4}$ and CHAODONG ZHANG ${ }^{1}$ \\ ${ }^{1}$ Department of Neurology, The First Affiliated Hospital of China Medical University, Shenyang, Liaoning 110001;
${ }^{2}$ Department of Neurology, The $463^{\text {th }}$ Hospital of the Chinese People's Liberation Army, Shenyang, Liaoning $110042 ;$
${ }^{3}$ Department of Clinical Pharmacy, China Medical University, Shenyang, Liaoning 110001;
${ }^{4}$ Department of Neurology, Shenyang First People's Hospital, Shenyang, Liaoning 110041, P.R. China
}

Received April 17, 2014; Accepted January 2, 2015

DOI: $10.3892 / \mathrm{mmr} .2015 .3421$

\begin{abstract}
Bone marrow stromal cells (BMSCs), derived from the mesoderm, have been applied in the repair and reconstruction of injured tissues. The present study was conducted to explore the effects of BMSCs on cell viability of tumor necrosis factor- $\alpha$ (TNF- $\alpha$ )-stimulated PC12 cells. PC12 cells were co-cultured with BMSCs under TNF- $\alpha$ treatment, with normal PC12 cells as controls. Results from an MTT assay indicated that BMSCs significantly increased cell growth and proliferation of TNF- $\alpha$-treated PC12 cells (survival rates were 56.71 and $76.86 \%$ for the positive control (PC) and co-culture group, respectively). Furthermore, Annexin V/propidium iodide staining and flow cytometric analysis demonstrated that TNF- $\alpha$ increased PC12-cell apoptosis from 3.49 to $40.74 \%$ in the negative control and $\mathrm{PC}$ group, and the apoptotic rate was significantly reduced upon co-culture with BMSCs to $16.97 \%$. In addition, data from reverse transcription-quantitative polymerase chain reaction and western blot analyses illustrated that TNF- $\alpha$-induced upregulation in TNF receptor (TNFR)-1 (TNFR1) and caspase-8 expression in PC12 cells were partially reversed by co-culture with BMSCs. In conclusion, the present study suggested that BMSCs protect PC12 cells against stimulation with TNF- $\alpha$, which is partially mediated through the TNFR/caspase signaling pathway. The results of the present study also suggested a therapeutic use of BMSCs in clinical neurodegenerative diseases.
\end{abstract}

Correspondence to: Dr Chaodong Zhang, Department of Neurology, The First Affiliated Hospital of China Medical University, 155 North Nanjing Street, Shenyang, Liaoning 110001, P.R China

E-mail: cmulhzhangchaodong@163.com

Key words: bone marrow stromal cells, PC12 cells, tumor necrosis factor- $\alpha$, proliferation, apoptosis

\section{Introduction}

Chronic progressive neurological diseases, or neurodegenerative diseases, including Parkinson's, Alzheimer's and Huntington's diseases, are characterized by irreversible loss of neuronal function in specific areas, apoptosis and death. Previous studies have demonstrated that neurodegenerative diseases are inextricably correlated with neuronal inflammation, such as tumor necrosis factor- $\alpha$ (TNF- $\alpha)$-induced neuronal injury $(1,2)$. At present, in spite of the progress which previous studies have made in the development of therapeutic strategies for neurodegenerative diseases $(3,4)$, it is necessary to further elucidate the underlying pathogenesis and to develop improved treatments.

Bone marrow stromal cells (BMSCs) are derived from the mesoderm and are able to undergo self-renewal and differentiation (5). Previous studies have demonstrated that BMSCs have the potential to differentiate into neuronal cells and that transplanted BMSCs are able to promote neuronal regeneration (6-8). However, the mechanisms of BMSCs in inflammatory factor-induced neuronal cell injuries remains to be fully elucidated.

In the present study, apoptosis was induced in PC12 cells by TNF- $\alpha$. PC12 cells were co-cultured with BMSCs using Transwell chambers in order to investigate whether BMSCs protect PC1 2 cells against TNF- $\alpha$ stimulation. Furthermore, the present study aimed to elucidate the effects of co-culture with BMSCs on the TNF receptor (TNFR)/caspase signaling pathway, which is associated with cell viability.

\section{Materials and methods}

Isolation and culture of BMSCs. Three Sprague-Dawley (SD) rats (mean body weight, $150 \mathrm{~g}$; 4-6 weeks-old) were purchased from the Animal Center of China Medical University (Shenyang, China). Animal experiments in the present study were approved by the ethical committee of China Medical University. SD rats were anesthetized via intraperitoneal injection of $10 \%$ chloral hydrate (Sinopharm Chemical Reagent Co., Ltd., Shanghai, 
China; $3.5 \mathrm{ml} / \mathrm{kg}$ body weight). Whole bone marrow was isolated from SD rats, as described previously (9), and cells were isolated and seeded in a $25-\mathrm{cm}^{2}$ plastic bottle at a concentration of $10^{6}$ cells $/ \mathrm{ml}$ in low-glucose Dulbecco's modified Eagle's medium (DMEM; GE Healthcare Life Sciences, Little Chalfont, UK) containing $20 \%$ fetal bovine serum (FBS; GE Healthcare Life Sciences). When adherent cells had reached $80 \%$ confluence, they were detached using $0.2 \%$ trypsin (GE Healthcare Life Sciences) and re-plated at a 1:2 ratio for continued passaging. Cells from passage 3 were cultured in low-glucose DMEM containing 5\% FBS.

BMSC identification. Cells were seeded onto sterilized cover slips, washed three times with phosphate-buffered saline (PBS; GE Healthcare Life Sciences) and fixed in 4\% formaldehyde (Sinopharm Chemical Reagent Co., Ltd.) for 15-20 min. Subsequent to washing three times for $2 \mathrm{~min}$ in PBS, the cells were blocked with $1 \%$ bovine serum albumin (Beijing Solarbio Science \& Technology Co., Ltd., Beijing, China) for $30 \mathrm{~min}$. Following blocking, DAPI (1:1,000; Roche Diagnostics, Basel, Switzerland) and fluorescently labeled mouse monoclonal antibodies: anti-CD44 (1:100; Cell Signaling Technology, Inc., Danvers, MA, USA) and anti-CD45 (1:50; BD Biosciences, Franklin Lakes, NJ, USA) were added. The samples were subsequently analyzed using a fluorescence microscope (LX70; Olympus Corporation, Tokyo, Japan).

Cell culture. Neuronal PC12 cells were purchased from the Cell Bank of Chinese Academy of Sciences (Shanghai, China). PC12 cells were cultured in RPMI 1640 medium (GE Healthcare Life Sciences) containing 10\% horse serum (Beijing Solarbio Science \& Technology Co., Ltd.) and 5\% FBS.

Cell treatment. PC12 cells were divided into three groups: i) Negative control group (NC), normal cultured PC12 cells; ii) positive control group (PC), PC12 cells treated with $50 \mathrm{ng} / \mathrm{ml}$ TNF- $\alpha$ (Peprotech, Inc., Rocky Hill, NJ, USA) for $2 \mathrm{~h}$, then removal of supernatant and addition of fresh serum-free DMEM for $24 \mathrm{~h}$; iii) co-culture group (co-culture), PC12 cells co-cultured with BMSCs using Transwell chambers (BD Biosciences), addition of $50 \mathrm{ng} / \mathrm{ml} \mathrm{TNF}-\alpha$ for $2 \mathrm{~h}$, removal of the supernatant and addition of fresh serum-free medium for $24 \mathrm{~h}$.

Cell proliferation assays. Cell proliferation was monitored using the MTT cell proliferation assay. In brief, PC12 cells and PC12 cells that had been co-cultured with BMSCs were transferred to 96-well plates and seeded at a density of 5,000 cells $(100 \mu \mathrm{l}) /$ well with eight repeats for each group. Twenty hours later, $10 \mu \mathrm{l}$ MTT $(5 \mathrm{mg} / \mathrm{ml})$ was added to each well. Following $4-\mathrm{h}$ incubation at $37^{\circ} \mathrm{C}$, the medium was removed and dimethyl sulfoxide (Beijing Solarbio Science \& Technology Co., Ltd.) was added in order to dissolve the formazan crystals. Subsequently, the absorbance was measured at a wavelength of $570 \mathrm{~nm}$ with an ELISA microplate reader (Sunrise $^{\mathrm{TM}}$; Tecan Group Ltd., Männedorf, Switzerland). The percentage of cell proliferation was calculated according to the following formula: Survival rate $(\%)=\{$ [treated group optical density $(\mathrm{OD})_{570^{-}}$blank group $\left.\mathrm{OD}_{570}\right] /$ (control group $\mathrm{OD}_{570}$ - blank group $\left.\left.\mathrm{OD}_{570}\right)\right\} \times 100 \%$.
Table I. Oligonucleotide primer sets for reverse transcription-quantitative polymerase chain reaction.

\begin{tabular}{llc}
\hline $\begin{array}{l}\text { Gene } \\
\text { symbol }\end{array}$ & \multicolumn{1}{c}{$\begin{array}{c}\text { Sequence } \\
\left(5^{\prime}-3^{\prime}\right)\end{array}$} & $\begin{array}{c}\text { Product size } \\
(\mathrm{bp})\end{array}$ \\
\hline TNFR1-F & CGGGCTTACTGGATACGA & 143 \\
TNFR1-R & GCAACGCTGGTGAATGAA & \\
TNFR2-F & CACCTGTCTCGTCCTACCT & 333 \\
TNFR2-R & AACAACTGGGCTCCTCTAA & \\
$\beta$-actin-F & CGTGCGTGACATTAAAGAG & 132 \\
$\beta$-actin-R & TTGCCGATAGTGATGACCT & \\
\hline
\end{tabular}

TNFR1, tumor necrosis factor receptor-1; bp, base pairs.

Apoptosis analysis. The Annexin V-fluorescein isothiocyanate (FITC) Apoptosis Detection kit (Nanjing KeyGen Biotech. Co., Ltd., Nanjing, China) was used to assess the apoptosis of PC12 cells in each group. PC12 cells were harvested subsequent to digestion with trypsin, washed twice with PBS and then re-suspended in $300 \mu 1$ Annexin V-FITC binding buffer. Cells were incubated with $10 \mu \mathrm{l}$ Annexin V-FITC for $10 \mathrm{~min}$ and $5 \mu \mathrm{l}$ propidium iodide (PI) for $5 \mathrm{~min}$. Finally, $200 \mu \mathrm{l}$ binding buffer was added and the samples were incubated for $15 \mathrm{~min}$ at room temperature in the dark and then subjected to flow cytometric analysis (BD FACSCalibur; BD Biosciences).

Analysis of morphological alterations. PC12 cells were harvested following digestion with $0.25 \%$ trypsin, washed once with PBS and then fixed with $4 \%$ glutaraldehyde (Sinopharm Chemical Reagent Co., Ltd.) for 12 h. Subsequent to washing three times with $\mathrm{PBS}$ at $4^{\circ} \mathrm{C}$, the samples were fixed with $1 \%$ osmium tetroxide (J\&K Scientific Ltd., Beijing, China) for 15-30 min. Subsequently, the samples were dehydrated using acetone (Sinopharm Chemical Reagent Co., Ltd.) and embedded in epon resin. Following cutting semi-thin sections $(50 \mathrm{~nm})$ and staining with lead citrate (J\&K Scientific Ltd., Beijing, China) and uranyl acetate (Micxy Reagent, Chengdu, China), the sections were analyzed by transmission electron microscopy (TEM; H-7100; Hitachi Ltd., Tokyo, Japan).

Reverse transcription-quantitative polymerase chain reaction (RT-qPCR). Total RNA was isolated using TRIzol (Takara Biotechnology, Co., Ltd., Dalian, China) according to the manufacturer's instructions. The concentration and purity of RNA were determined by detecting the absorbance at wavelengths at 260 and $280 \mathrm{~nm}$ (NANO 2000; Thermo Fisher Scientific, Waltham, MA, USA). An equal amount of RNA was reverse transcribed to synthesize complementary DNA (cDNA) using a PrimeScript RT reagent kit (Takara Biotechnology, Co., Ltd.). RT-qPCR was conducted using SYBR-Green (Takara Biotechnology, Co., Ltd.) on a LightCycler 480 PCR system (Roche Diagnostics). The sequences of the primers are presented in Table I. $\beta$-actin was used as the endogenous RNA control to normalize samples. Data were analyzed by the comparative threshold cycle method. 
A

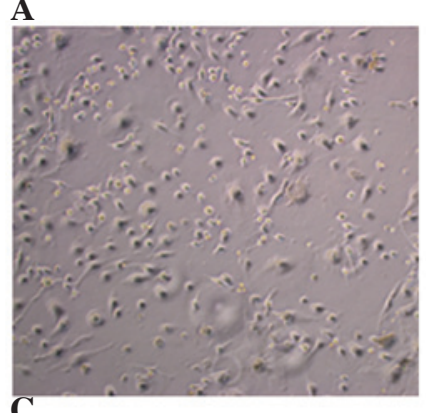

B
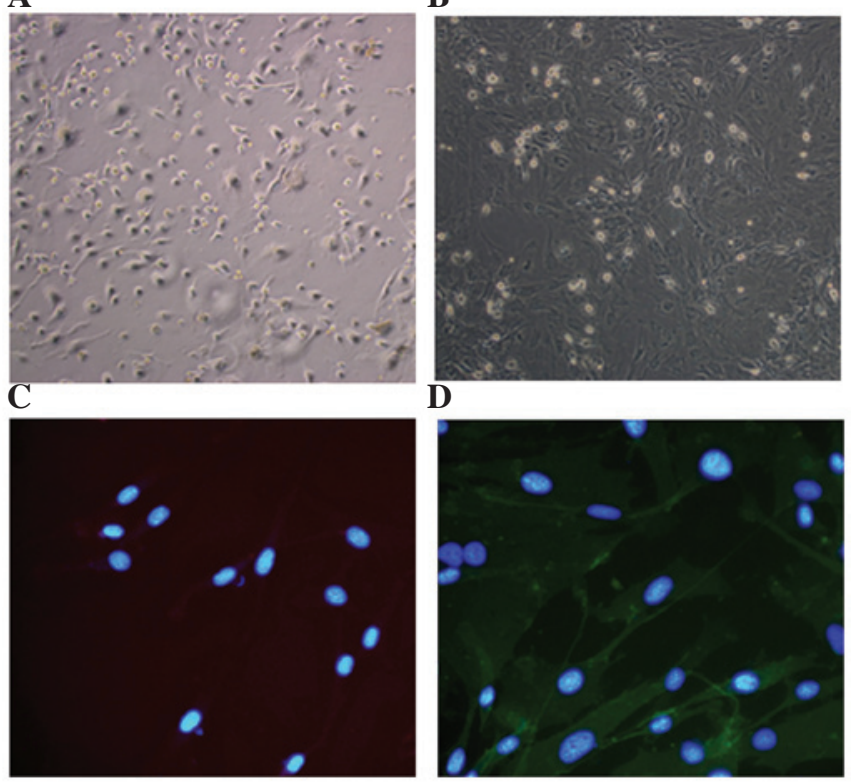

Figure 1. Identity and purity of BMSCs. (A) BMSCs appeared circular under the microscope and had grown in the medium at the second day (magnification, x100. (B) Passage cultured BMSCs presented with a shuttle- and fibroblast-like shape (magnification, $\mathrm{x} 100$ ). BMSCs were observed to be (C) negative for the CD45 antigen (magnification, $\mathrm{x} 400$ ) and (D) positive for the CD44 antigen (magnification, $\mathrm{x} 400$ ). Green fluorescence, CD44 antigen: blue fluorescence, DAPI. BMSCs, bone marrow stromal cells.

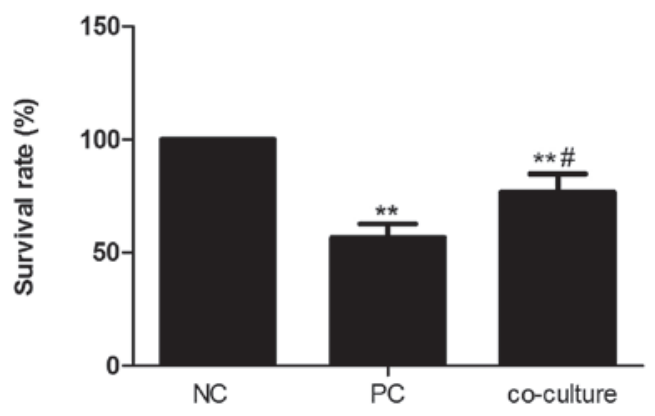

Figure 2. Effect of PC12 cell proliferation induced by TNF- $\alpha$ and BMSCs. PC12 cells were stimulated with $50 \mathrm{ng} / \mathrm{ml} \mathrm{TNF}-\alpha$ and cultured with BMSCs in the co-culture group. PC12 cell proliferation was determined by the MTT assay. Values are presented as the mean \pm standard deviation. " $\mathrm{P}<0.05 \mathrm{vs.} \mathrm{NC}$; ${ }^{* *} \mathrm{P}<0.01$ vs. NC; ${ }^{\text {"P }}<0.05$ vs. PC. TNF- $\alpha$, tumor necrosis factor- $\alpha$; BMSCs, bone marrow stromal cells; NC, negative control; PC, positive control.

Western blot analysis. Cells were washed with cold PBS and subsequently harvested using lysis buffer (Beyotime Institute of Biotechnology, Haimen, China). Following incubation on ice for $40 \mathrm{~min}$, the supernatant was centrifuged at $12,000 \mathrm{x} \mathrm{g}$ for $20 \mathrm{~min}$ at $4^{\circ} \mathrm{C}$ and the concentrations of these protein samples were determined using the Lowry method (10). Subsequently, proteins were denatured in sample buffer (Beijing Solarbio Science \& Technology Co., Ltd.) for $5 \mathrm{~min}$ at $100^{\circ} \mathrm{C}$. Equal amounts of proteins were loaded onto each lane of $8 \%$ SDS-PAGE gel and were then transferred onto polyvinylidene fluoride membranes (EMD Millipore, Billerica, MA, USA). The membranes were blocked with 5\% non-fat dry milk (Yili, Inner Mongolia, China) in Tris-buffered saline (TBS) and then incubated with rabbit polyclonal anti-caspase-8 (1:400; BioVision, Inc., Milpitas, CA, USA) and mouse monoclonal $\beta$-actin (1:10,000; Kangchen, Shanghai, China) antibodies at $4^{\circ} \mathrm{C}$ overnight. Subsequent to washing with TBS supplemented with $0.1 \%$ Tween-20, the membranes were incubated with secondary antibodies (1:2,000) for $2 \mathrm{~h}$. Densitometric analysis was conducted with ChemiImager 5500 version 2.03 (ProteinSimple, Santa Clara, CA, USA).

Statistical analysis. Values are presented as the mean \pm standard deviation. Data were analyzed using a one-way analysis of variance. All statistical analyses were conducted using SPSS version 13.0 software (SPSS, Inc., Chicago, IL, USA). $\mathrm{P}<0.05$ was considered to indicate a statistically significant difference.

\section{Results}

Culture and identification of BMSCs. Isolation and primary culture of BMSCs were performed as described. BMSCs appeared circular under the microscope and grew efficiently in the medium at the second day (Fig. 1A). Subsequent to passage cultures, the BMSCs appeared to have a shuttle- and fibroblast-like morphology (Fig. 1B). To assess the purity of the isolated cells, immunofluorescence staining was conducted and the results demonstrated that the majority of cells were CD45-negative and CD44-positive (Fig. 1C and D). It was observed that the BMSCs obtained in the present study were highly pure.

BMSCs reverse the TNF- $\alpha$-induced decrease in PC12 cell survival rate. To assess the effect of BMSCs on the viability of PC1 2 cells treated with TNF- $\alpha$, the proliferation of PC12 cells in each group was assessed using an MTT assay. As presented in Fig. 2, TNF- $\alpha$ treatment significantly reduced the proliferation of PC12 cells $(\mathrm{P}<0.01)$ and the previous low survival rate of PC12 cells was significantly improved by co-culture with BMSCs (56.71 and $76.86 \%$ in the PC and co-culture groups, respectively; $\mathrm{P}<0.05)$. These results suggested that $\mathrm{BMSCs}$ significantly increased the low survival rate of PC12 cells stimulated with TNF- $\alpha$.

BMSCs inhibit TNF- $\alpha$-induced apoptosis of PC12 cells. Cell apoptosis is known to be a key factor in the pathogenesis of neurodegenerative diseases. As cell proliferation was observed, an Annexin V/PI (AV/PI) staining method was used followed by flow cytometric analysis. The results demonstrated that TNF- $\alpha$ induced significant apoptosis in PC12 cells and BMSCs $(\mathrm{P}<0.001)$, notably suppressing apoptosis in PC12 cells (Fig. 3A). The percentage of apoptotic cells was $3.49 \%$ in the control group, whereas it was 40.74 and $16.97 \%$ in the PC and co-culture groups, respectively. Furthermore, TEM was used to detect the morphological alterations in PC12 cells in each group. Fig. 3B illustrates that the normal PC12 cells were round, containing predominantly euchromatin in the cell nucleus. In the PC group, PC12 cells exhibited TNF- $\alpha$-induced ultrastructural alterations that were characteristic of apoptosis, including cytoplasmic vacuolation and chromosome condensation at the periphery of the nucleus. These morphological markers of apoptosis were largely abolished by treatment with BMSCs. These observations suggested that TNF- $\alpha$-induced apoptosis in PC12 cells was diminished by co-culture with BMSCs. 

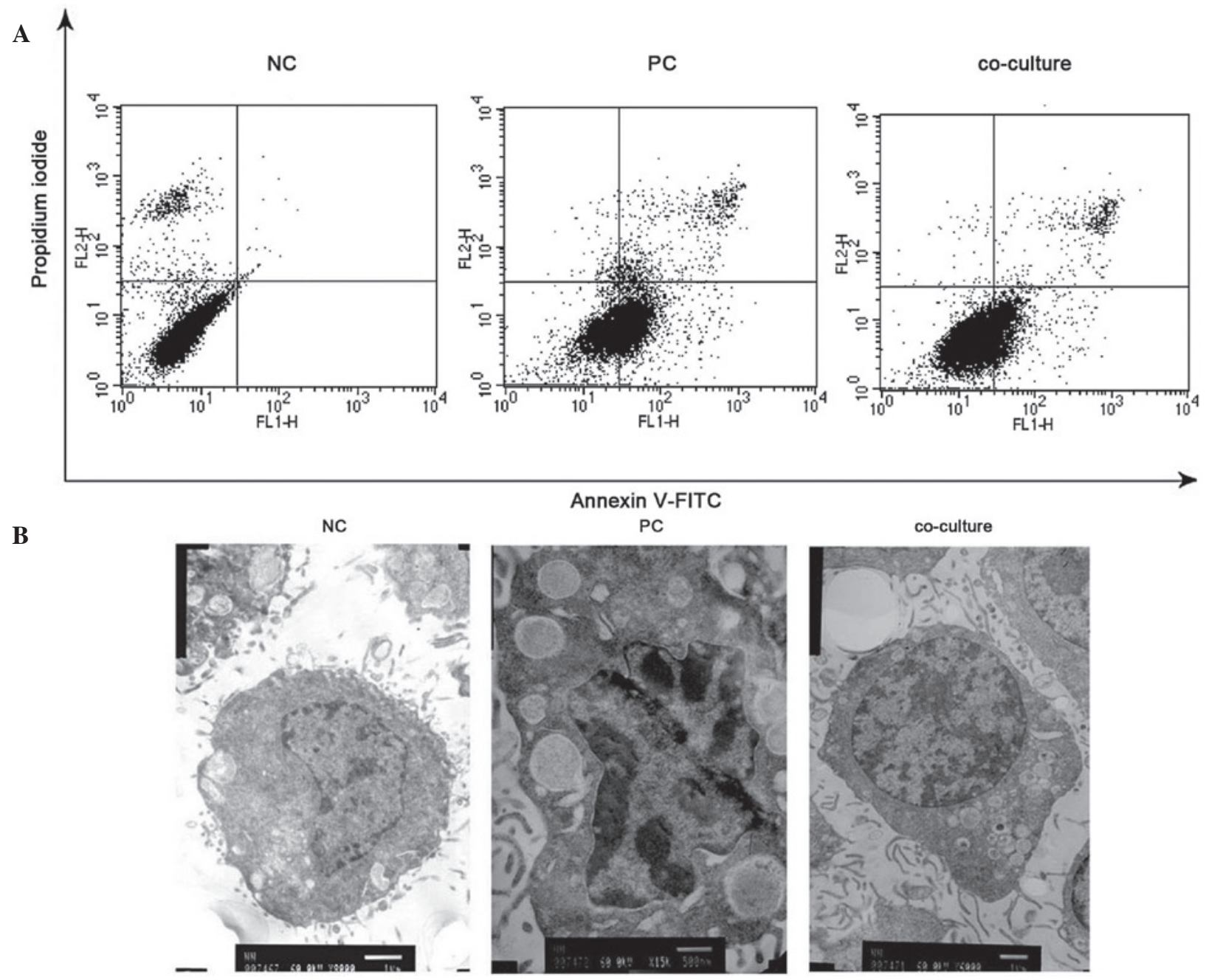

Figure 3. Effect of BMSCs on PC12 cell apoptosis induced by TNF- $\alpha$. (A) AV/PI staining for assessment of apoptosis. PC12 cells were harvested, stained with $\mathrm{AV} / \mathrm{PI}$ and then subjected to flow cytometric analysis. (B) TEM for analysis of morphological alterations. PC12 cells were harvested, fixed with $1 \%$ osmium tetroxide, stained with lead citrate and uranyl acetate and then examined by TEM (NC, magnification x8,000; PC, magnification x15,000; co-culture, magnification x6,000). BMSCs, bone marrow stromal cells; TNF- $\alpha$, tumor necrosis factor- $\alpha$; AV/PI, Annexin V/propidium iodide; TEM, transmission electron microscopy; NC, negative control; PC, positive control.

BMSCs interact with PC12 cells via the TNFR/caspase signaling pathway. To investigate the signaling pathway activated by BMSCs in PC12 cells, the expression levels of TNFR1, TNFR2 and caspase-8 were examined. As presented in Fig. 4A, the mRNA levels of TNFR1 were significantly increased in the $\mathrm{PC}$ group compared with those in the $\mathrm{NC}$ group $(\mathrm{P}<0.05)$. Of note, this induction of TNFR1 mRNA expression by TNF- $\alpha$ was significantly inhibited in the presence of BMSCs $(\mathrm{P}<0.05)$. However, the mRNA levels of TNFR2 were observed to be affected in a different way to those of TNFR1, as they increased in the presence of BMSCs. Furthermore, treatment with TNF- $\alpha$ significantly increased the levels of caspase- 8 protein expression, which was markedly attenuated following co-incubation with BMSCs (Fig. 4B and C). These results suggested that the TNFR/caspase signaling pathway was involved in the alteration of cell apoptosis induced by TNF- $\alpha$.

\section{Discussion}

The slow and progressive loss and dysfunction of axons and neurons is commonly observed in the central nervous system (CNS) during the pathogenesis of neurodegenerative diseases (11). PC12 cells are commonly used in models of neuronal differentiation in neuropharmacological and neurophysiological studies (12). PC12 is a cell line derived from a pheochromocytoma of the rat adrenal medulla and has similar characteristics to those of neuroendocrine cells. Although neuronal degeneration predominantly starts with specific neuronal populations, there are various similarities between different neurodegenerative diseases. These include atypical protein oligomerization and assemblies in addition to induced cell apoptosis and death. In addition, previous studies indicated that inflammatory cytokines, such as TNF- $\alpha$, may be associated with neuronal cell death and neurodegenerative disorders (13). Furthermore, TNF- $\alpha$ has been shown to be associated with glutamate-induced neuronal cell death (14), and is able to induce neurotoxicity through glutamate production (15). In the present study, the function of BMSCs in proliferation and apoptosis of PC12 cells treated with TNF- $\alpha$ was investigated. The results indicated that BMSCs inhibited the TNF- $\alpha$-induced low survival rate and apoptosis of PC12 cells. Furthermore, it was suggested 
A
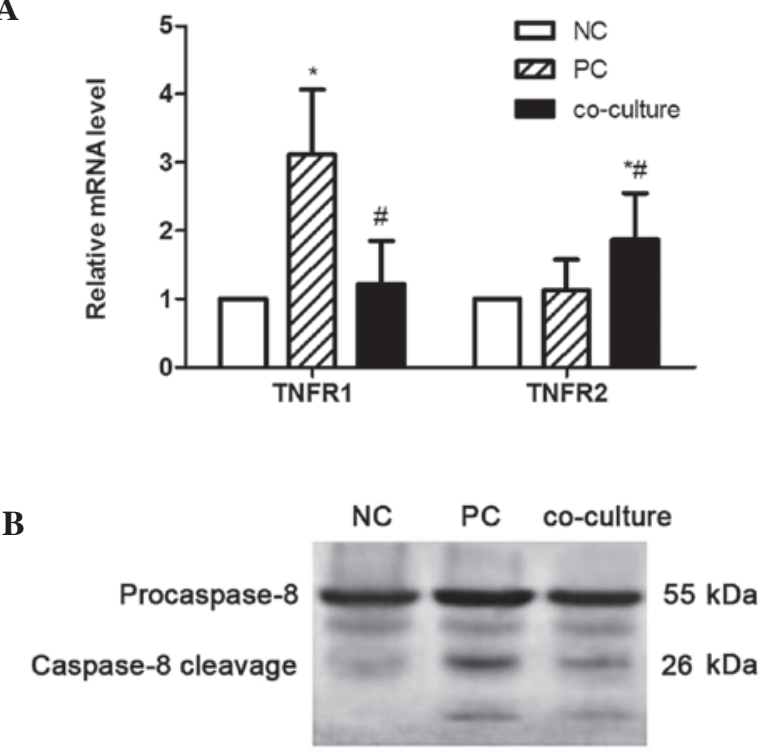

$\beta$-actin
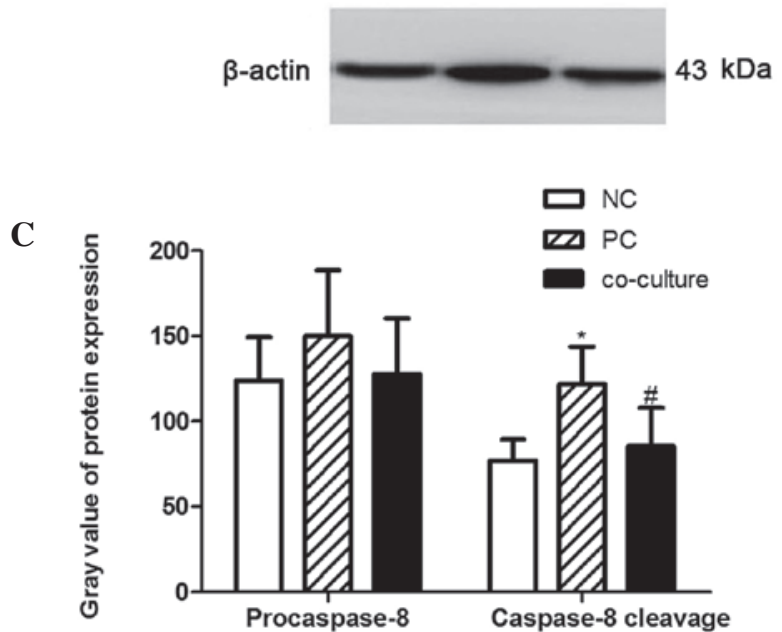

Figure 4. Effect of BMSCs on the expression of TNFR1, TNFR2, procaspase- 8 and caspase- 8 in TNF- $\alpha$-treated PC12 cells. PC12 cells were cultured with TNF- $\alpha(50 \mathrm{ng} / \mathrm{ml})$ in the absence or presence of BMSCs (A) RT-qPCR was used for analysis of TNFR1 and TNFR2 mRNA expression. (B) Representative western blots for detection of procaspase- 8 and caspase- 8 with the protein size expressed in $\mathrm{kDa}$. (C) Protein levels were normalized to $\beta$-actin and data were subjected to densitometric quantification. Values are presented as the mean \pm standard deviation. ${ }^{*} \mathrm{P}<0.05$ vs. $\mathrm{NC}$ ${ }^{\#} \mathrm{P}<0.05$ vs. PC. BMSCs, bone marrow stromal cells; TNFR1, tumor necrosis factor receptor 1 ; RT-qPCR, reverse transcription-quantitative polymerase chain reaction; $\mathrm{NC}$, negative control; $\mathrm{PC}$, positive control.

that the TNFR/caspase signaling pathway was involved in these processes.

BMSCs are important candidates for therapeutic use in the repair of injured tissue, as they possess multilineage differentiation potential (16). The primary cultured BMSCs were isolated from SD rats in the present study according to the method of a previous study (9). Subcultured BMSCs exhibited a shuttle- and fibroblast-like shape. In addition, immunofluorescence results demonstrated that the BMSCs expressed CD44 but not CD45, which is in agreement with a previous study by Herzog et al (17), and the BMSCs obtained were considered to be highly pure. Previous studies have described the effects of BMSCs on neurodegenerative diseases; for example, Koh et al (18) demonstrated that the functional deficiency of BMSCs in patients with amytrophic lateral sclerosis is proportional to the rate of disease progression. Sun et al (19) identified that BMSCs induced by tricyclodecane-9-yl-xanthogenate differentiated into cholinergic neuron-like cells, which were able to promote functional recovery and neural protein following spinal cord injury. In addition, Pastor et al (20) reported that glial cell line-derived neurotrophic factor expression was upregulated in the spinal cords of mice treated with BMSCs. These studies indicated that BMSCs not only differentiate into neuron-like cells, but also secrete neurotrophic factor and create a suitable environment for neural cell survival.

Through inflammatory signals from the CNS and through removal of neurotoxic proteins and damaged tissue, the CNS returns to its normative state; however, neuroinflammation which is not inhibited and repaired may lead to cellular dysfunction and a reduction in neuronal dendritic branching, and result in neurodegenerative disease $(21,22)$. To assess the potential role of BMSCs in regulating the number of $\mathrm{PC} 12$ cells induced by TNF- $\alpha$, an MTT assay was performed in order to detect the proliferation of $\mathrm{PC} 12$ cells in each group. The analysis demonstrated that TNF- $\alpha$ inhibited the proliferation of PC12 cells and resulted in a lower survival rate of $\mathrm{PC} 12$ cells compared with that of the NC group. The addition of $50 \mathrm{ng} / \mathrm{ml}$ TNF- $\alpha$ significantly inhibited the proliferation of PC12 cells, and this inhibition was alleviated by BMSCs. Furthermore, co-culture with BMSCs resulted in an improvement of the low survival rate induced by TNF- $\alpha$. TNF- $\alpha$ is a cytokine involved in systemic inflammation and was first described by Carswell et al in 1975 (23). Previous studies have suggested that TNF- $\alpha$ is associated with neuronal maturation and arborization. Golan et al (24) observed that a lack of TNF- $\alpha$ resulted in accelerated dentate gyrus development, which correlated to increased levels of nerve growth factor. Neumann et al (25) indicated that treatment with TNF- $\alpha$ led to a reduction in dendritic branching. In addition, inhibition of neuronal cell death and promotion of regeneration by BMSCs had also been observed in a previous study (26). Observations of the present study suggested that BMSCs significantly suppress TNF- $\alpha$-induced inhibition of proliferation in PC12 cells.

Apoptosis of neuronal cells is a key contributor in the pathogenesis of neurodegeneration and the inhibition of neuronal cell apoptosis is important for the treatment of neurodegenerative diseases $(27,28)$. The aim of the present study was to investigate the anti-neurodegenerative mechanism of BMSCs in PC12 cells against TNF- $\alpha$ treatment, as TNF- $\alpha$ acts as a stimulator in the pathogenesis of neurodegenerative disease. In agreement with previous studies, the results of the present study suggested that TNF- $\alpha$ significantly induced PC12 cell apoptosis $(21,29)$. In addition, it was observed in the present study that BMSCs attenuated TNF- $\alpha$-induced PC1 2 cell apoptosis, as demonstrated by AV/PI staining and TEM. These results are consistent with those of a previous study by Guo et al (30), which indicated that BMSCs inhibit cisplatin- and perimenopause-induced rat granulosa cell apoptosis via a $\mathrm{p} 21-$, B-cell lymphoma-associated $\mathrm{X}$ protein and c-myc- dependent apoptotic pathway. The results of the present study, together with those of previous studies, suggested that BMSCs may be useful for the treatment of neurodegenerative diseases.

The binding of TNF- $\alpha$ to its receptor TNFR1 is able to induce cell survival, apoptosis or death through the 
formation of two sequential complexes. Complex I triggers activation of the transcription factor nuclear factor- $\kappa \mathrm{B}$ and prevents caspase- 8 activation; however, complex II leads to caspase-dependent apoptosis (31-33). More specifically, when PC12 cells were stimulated with TNF- $\alpha$, the TNF receptor-associated death domain, which recruits TNFR1, can bind to the Fas-associated death domain via a homologous sequence at the $\mathrm{C}$-terminus. These binding proteins that formed complex II can facilitate the oligomerization of pro-caspase- 8 and then auto-cleave into activated caspase- 8 , eventually resulting in a cascade leading to apoptosis $(34,35)$. In the present study, the mRNA levels of TNFR1 and the protein levels of caspase- 8 were increased in the PC group; however, treatment with BMSCs inhibited the increase induced by TNF- $\alpha$ in TNFR 1 and caspase- 8 in the co-culture group. By contrast, the TNFR2 mRNA levels were elevated in the group co-cultured with BMSCs. Thus, it is suggested that BMSCs are able to inhibit TNFR1 to recruit caspase- 8 and ultimately suppress the apoptosis of PC12 cells. However, further studies are required to elucidate the precise mechanisms by which BMSCs exert anti-apoptotic effects in TNF- $\alpha$-stimulated PC12 cells.

In conclusion, the results of the present study demonstrated that BMSCs effectively suppress TNF- $\alpha$-induced proliferation inhibition and apoptosis in cultured PC12 cells. In addition, the TNFR/caspase signaling pathway was involved in the alterations of apoptosis and proliferation induced by TNF- $\alpha$. The observations of the present study also implied that BMSCs possess therapeutic potential in the prevention of neurodegenerative diseases and that their application presents a promising therapeutic strategy.

\section{References}

1. Glass CK, Saijo K, Winner B, Marchetto MC and Gage FH: Mechanisms underlying inflammation in neurodegeneration. Cell 140: 918-934, 2010.

2. Amor S, Puentes F, Baker D and van der Valk P: Inflammation in neurodegenerative diseases. Immunology 129: 154-169, 2010.

3. Deierborg T, Soulet D, Roybon L, Hall V and Brundin P: Emerging restorative treatments for Parkinson's disease. Prog Neurobiol 85: 407-432, 2008 .

4. Zhao J and Xu Q: Emerging restorative treatments for Parkinson's disease: Manipulation and inducement of dopaminergic neurons from adult stem cells. CNS Neurol Disord Drug Targets 10: 509-516, 2011

5. Mezey E: The therapeutic potential of bone marrow-derived stromal cells. J Cell Biochem 112: 2683-2687, 2011.

6. Cho H, Seo YK, Yoon HH, et al: Neural stimulation on human bone marrow-derived mesenchymal stem celels by extremely low frequency electromagnetic fields. Biotechnol Prog 28: 1329-1335, 2012.

7. Tang Y, Cui YC, Wang XJ, et al: Neural progenitor cells derived from adult bone marrow mesenchymal stem cells promote neuronal regeneration. Life Sci 91: 951-958, 2012.

8. Polisetti N, Chaitanya VG, Babu PP and Vemuganti GK: Isolation, characterization and differentiation potential of rat bone marrow stromal cells. Neurol India 58: 201-208, 2010.

9. Zhao T, Yan W, Xu K, Qi Y, Dai X and Shi Z: Combined treatment with platelet-rich plasma and brain-derived neurotrophic factor-overexpressing bone marrow stromal cells supports axonal remyelination in a rat spinal cord hemi-section model. Cytotherapy 15: 792-804, 2013.

10. Waterborg JH and Matthews HR: The Lowry method for protein quantitation. Methods Mol Biol 32: 1-4, 1994.

11. Varvel NH, Bhaskar K, Patil AR, Pimplikar SW, Herrup K and Lamb BT: Abeta oligomers induce neuronal cell cycle events in Alzheimer's disease. J Neurosci 28: 10786-10793, 2008.
12. Jung IS, Kim HJ, Noh R, Kim SC and Kim CW: Effects of extremely low frequency magnetic fields on NGF induced neuronal differentiation of $\mathrm{PC} 12$ cells. Bioelectromagnetics 35: 459-469, 2014

13. Al-Gayyar MM and Elsherbiny NM: Contribution of TNF- $\alpha$ to the development of retinal neurodegenerative disorders. Eur Cytokine Netw 24: 27-36, 2013.

14. Kogo J, Takeba Y, Kumai T, et al: Involvement of TNF-alpha in glutamate-induced apoptosis in a differentiated neuronal cell line. Brain Res 1122: 201-208, 2006

15. Ye L, Huang Y, Zhao L, et al: IL-1 $\beta$ and TNF- $\alpha$ induce neurotoxicity through glutamate production: a potential role for neuronal glutaminase. J Neurochem 125: 897-908, 2013.

16. Su YR, Wang J, Wu JJ, Chen Y and Jiang YP: Overexpression of lentivirus-mediated glial cell line-derived neurotrophic factor in bone marrow stromal cells and its neuroprotection for the PC12 cells damaged by lactacystin. Neurosci Bull 23: 67-74, 2007.

17. Herzog EL, Chai L and Krause DS: Plasticity of marrow-derived stem cells. Blood 102: 3483-3493, 2003.

18. Koh SH, Baik W, Noh MY, et al: The functional deficiency of bone marrow mesenchymal stromal cells in ALS patients is proportional to disease progression rate. Exp Neurol 233: 472-480, 2012.

19. Sun C, Shao J, Su L, et al: Cholinergic neuron-like cells derived from bone marrow stromal cells induced by tricyclodecane-9-yl-xanthogenate promote functional recovery and neural protection after spinal cord injury. Cell Transplant 22: 961-975, 2013.

20. Pastor D, Viso-León MC, Jones J, et al: Comparative effects between bone marrow and mesenchymal stem cell transplantation in GDNF expression and motor function recovery in a motorneuron degenerative mouse model. Stem Cell Rev 8: 445-458, 2012.

21. Park KM and Bowers WJ: Tumor necrosis factor-alpha mediated signaling in neuronal homeostasis and dysfunction. Cell Signal 22: 977-983, 2010.

22. Paganelli R, Di Iorio A, Patricelli L, et al: Proinflammatory cytokines in sera of elderly patients with dementia: levels in vascular injury are higher than those of mild-moderate Alzheimer's disease patients. Exp Gerontol 37: 257-263, 2002.

23. Carswell EA, Old LJ, Kassel RL, Green S, Fiore N and Williamson B: An endotoxin-induced serum factor that causes necrosis of tumors. Proc Natl Acad Sci USA 72: 3666-3670, 1975.

24. Golan H, Levav T, Mendelsohn A and Huleihel M: Involvement of tumor necrosis factor alpha in hippocampal development and function. Cereb Cortex 14: 97-105, 2004.

25. Neumann H, Schweigreiter R, Yamashita T, Rosenkranz K, Wekerle $\mathrm{H}$ and Barde YA: Tumor necrosis factor inhibits neurite outgrowth and branching of hippocampal neurons by a rho-dependent mechanism. J Neurosci 22: 854-862, 2002.

26. Hsu SH, Kuo WC, Chen YT, et al: New nerve regeneration strategy combining laminin-coated chitosan conduits and stem cell therapy. Acta Biomater 9: 6606-6615, 2013.

27. Kermer P, Liman J, Weishaupt JH and Bähr M: Neuronal apoptosis in neurodegenerative diseases: from basic research to clinical application. Neurodegener Dis 1: 9-19, 2004.

28. Zhang H, Zhang YW, Chen Y, et al: Appoptosin is a novel pro-apoptotic protein and mediates cell death in neurodegeneration. J Neurosci 32: 15565-15576, 2012.

29. Wang C, Zhang Q, Qian Y and Zhao M: p,p'-DDE induces apoptosis through the modulation of tumor necrosis factor $\alpha$ in PC12 cells. Chem Res Toxicol 27: 507-513, 2014.

30. Guo JQ, Gao X, Lin ZJ, et al: BMSCs reduce rat granulosa cell apoptosis induced by cisplatin and perimenopause. BMC Cell Biol 14: 18, 2013.

31. Micheau O and Tschopp J: Induction of TNF receptor I-mediated apoptosis via two sequential signaling complexes. Cell 114: 181-190, 2003.

32. Schneider-Brachert W, Tchikov V, Neumeyer J, et al: Compartmentalization of TNF receptor 1 signaling: internalized TNF receptosomes as death signaling vesicles. Immunity 21 : 415-428, 2004

33. Schütze S, Tchikov V and Schneider-Brachert W: Regulation of TNFR1 and CD95 signalling by receptor compartmentalization. Nat Rev Mol Cell Biol 9: 655-662, 2008.

34. Bradley JR and Pober JS: Tumor necrosis factor receptor-associated factors (TRAFs). Oncogene 20: 6482-6491, 2001.

35. Badiola N, Malagelada C, Llecha N, et al: Activation of caspase- 8 by tumour necrosis factor receptor 1 is necessary for caspase- 3 activation and apoptosis in oxygen-glucose deprived cultured cortical cells. Neurobiol Dis 35: 438-447, 2009. 